\title{
Analyzing the Influence of Mobile Phone Use of Drivers on Traffic Flow Based on an Improved Cellular Automaton Model
}

\author{
Yao Xiao and Jing Shi \\ Institute of Transportation Engineering, Tsinghua University, Beijing 100084, China \\ Correspondence should be addressed to Jing Shi; jingshi@tsinghua.edu.cn
}

Received 13 November 2014; Accepted 18 January 2015

Academic Editor: Xiaohui Liu

Copyright (c) 2015 Y. Xiao and J. Shi. This is an open access article distributed under the Creative Commons Attribution License, which permits unrestricted use, distribution, and reproduction in any medium, provided the original work is properly cited.

\begin{abstract}
This paper aimed to analyze the influence of drivers' behavior of phone use while driving on traffic flow, including both traffic efficiency and traffic safety. An improved cellular automaton model was proposed to simulate traffic flow with distracted drivers based on the Nagel-Schreckenberg model. The driving characters of drivers using a phone were first discussed and a value representing the probability to use a phone while driving was put into the CA model. Simulation results showed that traffic flow rate was significantly reduced if some drivers used a phone compared to no phone use. The flow rate and velocity decreased as the proportion of drivers using a phone increased. While, under low density, the risk of traffic decreased first and then increased as the distracted drivers increased, the distracted behavior of drivers, like using a phone, could reduce the flow rate by 5 percent according to the simulation.
\end{abstract}

\section{Introduction}

Driving distractions have become a common phenomenon and one of the major factors connected with traffic crashes. Driver distraction means "a diversion of attention from driving, because driver is temporarily focused on event not related to driving" [1]. The most regular distractions while driving were using a mobile phone, conversing with passengers, eating and drinking, smoking, and other behaviors. As the frequency of mobile phone use increased, more and more drivers engaged with mobile phones while driving. An investigation in South of England revealed that 2.2\% of the drivers were using mobile phones while driving when observed from roadside, and the proportion of all kinds of distracted behaviors accounted for $14.4 \%$ [2]. The proportion of drivers using mobile phone while driving was very high, and approximately $43 \%-80 \%$ of drivers who owned a phone use it sometimes while driving [3-5]. Mobile phone use while driving should be paid more attention and its influence in traffic still needs to be studied.

The damage of mobile phone use while driving on traffic safety has been noticed by researchers. Back in 1996, the National Highway Traffic Safety Administration (NHTSA) has reported that about $25 \%$ of the crashes were related to distracted behaviors [6]. In 2007, a study which interviewed 1367 injured drivers in Australia suggested that driver distractions contributed to $14-33 \%$ of all serious traffic crashes [7]. An on-road research, in which the driver was observed by camera in car, reported that using a mobile phone while driving could make the driver 23 times more likely to have an accident [8]. Epidemiological research showed that using a mobile phone while driving could make the driver 4 times more likely to be injured in a traffic accident [9]. A number of studies have revealed how mobile phone use increased traffic accidents and hazarded the traffic safety, but the influence of driver distraction on traffic flow has not yet been studied. One of the major restricted factors was that field observation on traffic flow mixed with distracted driver' vehicle was nearly impossible. So the computer simulation was a good and feasible method to solve this problem. This paper will analyze the influence of mobile phone use on vehicle behavior and then simulate the traffic flow considering the mobile phone use of driver with CA model, trying to figure out how mobile phone use changes the traffic flow characteristics.

Cellular automata (CA) model was first applied into traffic flow simulation by Nagel and Schreckenberg in 1992 [10]. It is a dynamical system that is discrete in nature, which means that, in the model, road is divided into cells and 
time advances with discrete steps. Because of its efficiency and fast performance in simulation of traffic flow, a huge number of extended CA models were studied by the following researchers, such as for the impacts of tolling stations [11], the influence of intersections [12], the analysis of city traffic $[13,14]$, the effects of on-and-off ramps [15], mixed traffic flow with bicycles [16], and the influence of traffic lights [17]. Another advantage of CA model in current research is that as a microscopic traffic flow model, the behavior of single driver can be described through the behavior of individual vehicles.

This paper proposed an improved cellular automaton with stochastic variables which considered the driver behavior of mobile phone use, based on the basic NagelSchreckenberg model [18]. A two-lane highway traffic flow was simulated and the fundamental diagram obtained by CA model was discussed. The aim of this research is to achieve the characteristics of traffic flow when some of the drivers are using a mobile phone and figure out how this distraction behavior changes the features of traffic flow.

\section{Improved CA Model and Relevant Parameters}

2.1. Influence of Mobile Phone Use. Before presenting the improved CA model, the behavior of vehicle in which the driver used a mobile phone should be clarified first. Drivers who are using a mobile phone while driving may navigate at slower speed and keep longer distance from the vehicle in front [19], due to the diversion of attention. Driver chooses to slow down the vehicle when he is in conversation with the person on the phone, which makes the driver feel safer. In the meantime, it is an overloaded work for driver to follow the front car closely when he is in a dual task, so the space headway is also larger. Drivers will also reduce frequency of changing lanes when talking on a mobile phone and the whole time to finish the drive will be longer [20]. Different from other mixed traffic flow models, the driver does not maintain this special condition, that is, talking on a phone while driving. The driver may have a call at a certain time in the process of driving and continue to talk for several minutes and then end the conversation and resume normal driving.

2.2. Survey and Data Analysis. In order to get the characteristics of drivers using a phone while driving, a questionnaire survey entitled “Survey of Car Drivers' Distracted Driving Behavior in Beijing" was conducted via website among Beijing drivers. After removing the invalid questionnaires, 414 samples were obtained. Among the samples, 214 were female and 200 were male. The investigation displayed that $84.1 \%$ of the drivers might use a phone while driving at least once a week, and $29.9 \%$ of the drivers initiated a phone call while driving more than 5 times per week. It has become a common phenomenon and may become more frequent as the mobile phone use grows more and more popular. The investigation also showed that most of the drivers chose to keep the phone call short while driving. A 7-point Likert scale was used to estimate the length of time when driver talked on a phone while driving, where 1 is for very short and 7 for very long. Results showed that $69.6 \%$ of the drivers scored less than 4 points, meaning that they used a phone for relatively short time. $30.4 \%$ of the drivers scored 4 points or more, which means they still talked for a long time while driving.

2.3. Improved CA Model. A one-way two-lane highway is simulated, which is discretized into cells of $5 \mathrm{~m}$ length. In order to simulate different density of traffic flow, the highway is a $2 \mathrm{~km}$ long closed circle, which includes 400 cells in each lane. The state of each cell of the automata varies from -1 to 7 in the model, where state -1 refers to an unoccupied cell and states $0-7$ refer to cells occupied by a car of velocity 0 to velocity 7. That means a cell is either empty or occupied by a car. The states of the cells are updated every time step, which is 1 second, only depending on the present value of the cells of both lanes. The model runs according to the following rules and the value of the parameters in the model is explained in Section 2.3.

2.3.1. Distraction Conditions. The driver has two conditions: $D=1$ means using a phone while $D=0$ means not. If the driver in the vehicle is not using a mobile phone, he has the probability of $p_{\text {talk }}$ to start talking on a phone. And once a driver starts to talk, the conversation will last $T$ minutes; that is,

$$
\text { if } D=0 \text {, then } D=1 \text { with } p_{\text {talk }} \text {. }
$$

2.3.2. Changing Lane. Three rules must be satisfied before the vehicle chooses whether to change lane. The distance from the front car in the same lane, $d_{\text {front }}$, is smaller than the expected distance, $d_{\text {exp }}$, of the car. The distance from the front car in the neighbor lane, $d_{\text {neighbor }}$, is bigger than $d_{\text {front }}$ in the same lane. And the distance from the back car in the neighbor lane, $d_{\text {back }}$, is larger than $v_{\max }$, which is the maximum velocity of a $\operatorname{car}\left(v_{\max }=7\right)$. Even if all the three conditions are met, the vehicle just has the probability of $p_{\text {change }}$ to change lane. For the distracted driver, $p_{\text {change }}$ will be smaller because he prefers to maintain existing driving condition; that is,

$$
\begin{aligned}
& \text { if } d_{\text {front }}<d_{\text {exp }} \text { and } d_{\text {neighbor }}>d_{\text {front }} \text { and } d_{\text {back }}>v_{\text {max }} \text {, } \\
& \text { then change lane with } p_{\text {change }}
\end{aligned}
$$

2.3.3. Car Following. The process of car following includes three steps, acceleration, slowing down, and randomization. Firstly, the speed is increased by one, if it is lower than $v_{\max }$ and there is enough space ahead. Secondly, the speed must not be bigger than the distance from the front car. Thirdly, the speed decreases by one with probability $p_{\mathrm{dec}}$, if the speed is not zero. It should be noted that the vehicle which is determined to change lane is following the car ahead in the lane which it moves to. Moreover, the value of $v_{\max }$ and $p_{\mathrm{dec}}$ for distracted driver are different from normal driver; that is,

$$
\begin{aligned}
& \text { if } v<\text { gap, then } v=\min \left[v+1, v_{\max }\right] \text {; } \\
& \text { if } v>\text { gap, then } v=\min [v \text {, gap }] \text {; } \\
& \text { with } p_{\mathrm{dec}}, v=\max [v-1,0] \text {. }
\end{aligned}
$$




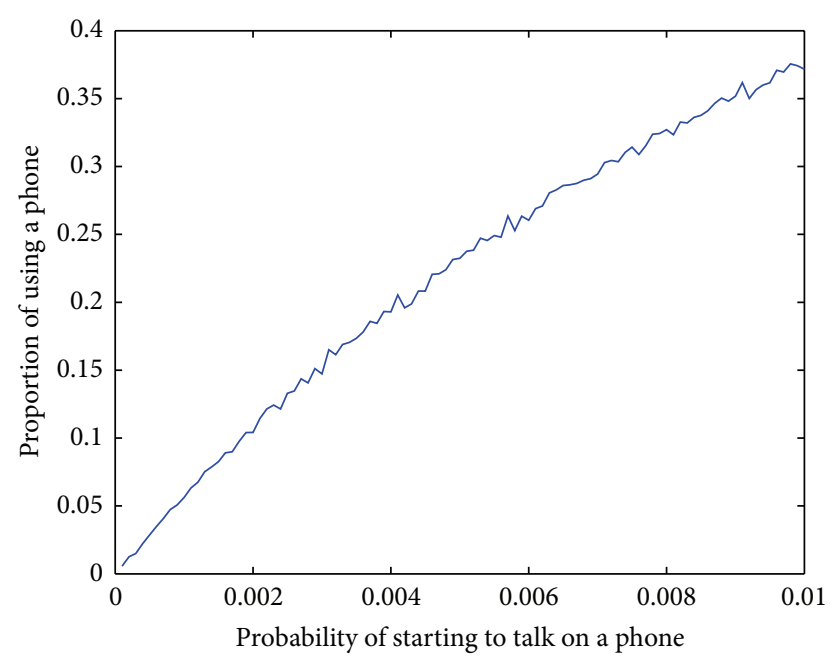

FIGURE 1: The relationship between $p_{\text {obser }}$ and $p_{\text {talk }}$.

\subsubsection{Car Propagation. Every car moves $v$ sites.}

2.4. Parameters. The first problem is how to decide the probability of starting to talk for a driver who is not using a phone, that is, $p_{\text {talk }}$. An investigation of drivers' real behavior with cameras installed in the vehicles found that the average time of conversation on a phone was about 1.5 minutes [21]. And, in this paper, it is assumed that every call of the driver lasts for 60 seconds. The result of observation indicates that $2.2 \%$ of the drivers are using a phone [2], but the relationship between indicator $p_{\text {obser }}$ and $p_{\text {talk }}$ is complex. Therefore, simulation was carried out to get the correlation between them. Given different value of $p_{\text {talk }}$, one thousand cars were modelling and the observed proportion of drivers who are using a phone at a certain time was calculated, as shown in Figure 1.

The proportion of using a phone increases very fast when $p_{\text {talk }}$ is smaller than $1 \%$. When the proportion is around $2 \%$, which is the actual situation, the probability of starting to talk on a phone is about $0.04 \%$.

Simon and Gutowitz [22] compared cellular automaton model with experimental data and found that the results of simulation were in close agreement with real-world traffic when $p_{\mathrm{dec}}$ is around 0.25 and $p_{\text {change }}$ is around 0.3. In our model, the maximum velocity of normal car is 7 and the values of $p_{\text {change }}$ and $p_{\text {dec }}$ are both 0.25 for the normal car. A driving simulator experiment showed that the average speed was lower, lane change frequency was lower, fluctuation in speed was higher, and the time to complete scenario was longer for drivers who are using a phone [23]. So, in this paper, $v_{\max }, p_{\text {change }}$, and $p_{\text {dec }}$ for drivers using a phone are 6 , 0.15 , and 0.35 . Every simulation lasts for 60 minutes and the first 30 minutes is warming time in order to make the flow stable.

\section{Results of Simulation and Discussion}

3.1. Mobile Phone Use and Traffic Flow. Assuming that the probability of starting to talk on a phone is $0.05 \%$,

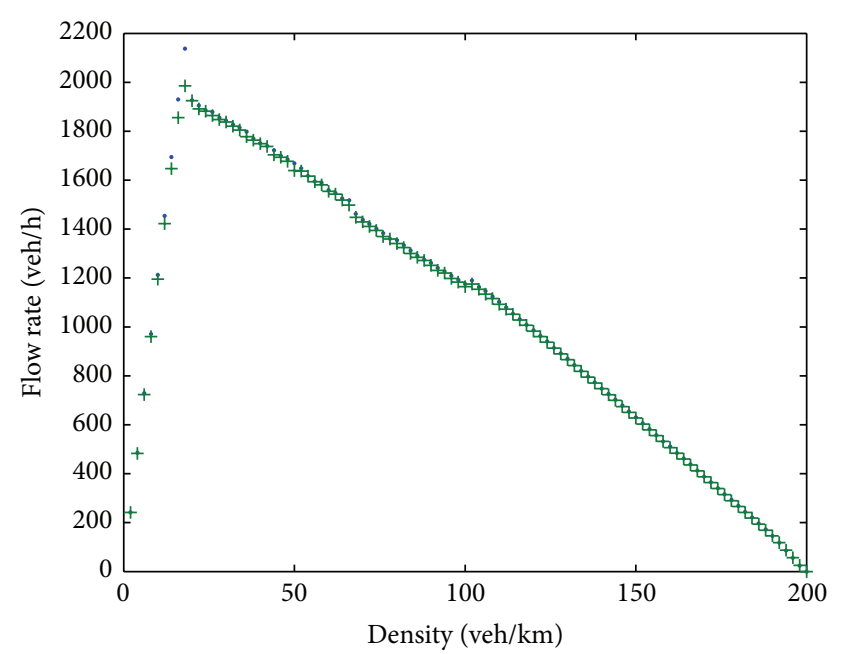

- No phone use

+ Probability of phone use $=0.05 \%$

FIgURE 2: The fundamental diagram of driving with phone use.

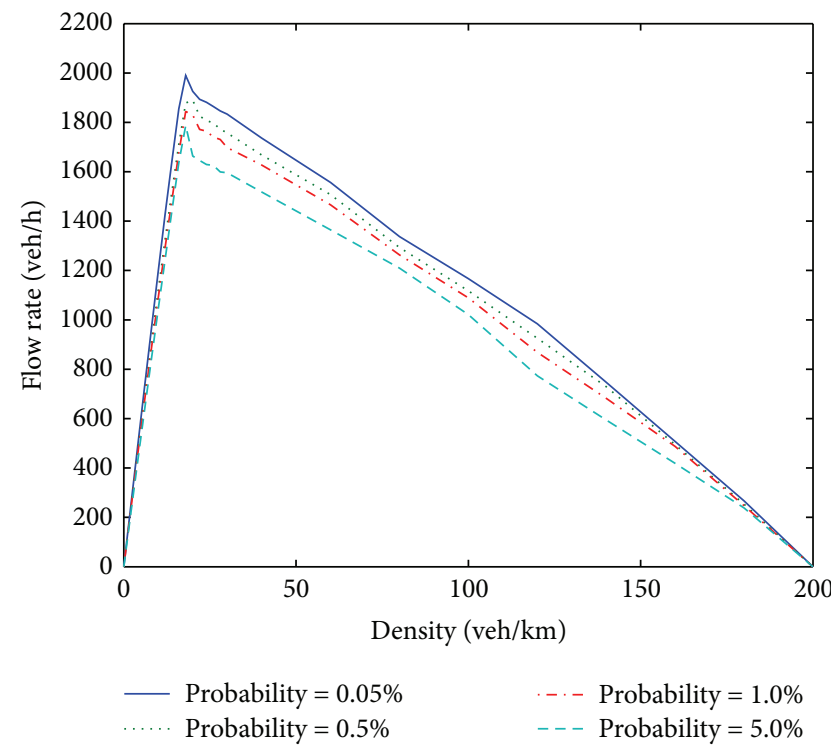

FIGURE 3: The fundamental diagram with different probability of phone use.

the fundamental diagram of two situations was simulated, no drivers using a phone and drivers using with this probability. The density of traffic flow is equivalent with the occupancy of cells. The cars are randomly distributed in the initial time and the simulation is on a circular road with periodic boundary conditions.

According to Figure 2, the flow rate of situation in which drivers may use a phone is apparently lower than normal situation, when density of vehicle is between 10 and $20 \mathrm{veh} / \mathrm{km}$. And there are no significant differences between two situations when density is higher than 20 or lower than $10 \mathrm{veh} / \mathrm{km}$. In the condition of free flow, the vehicle which is distracted will highly reduce the efficiency of traffic, 


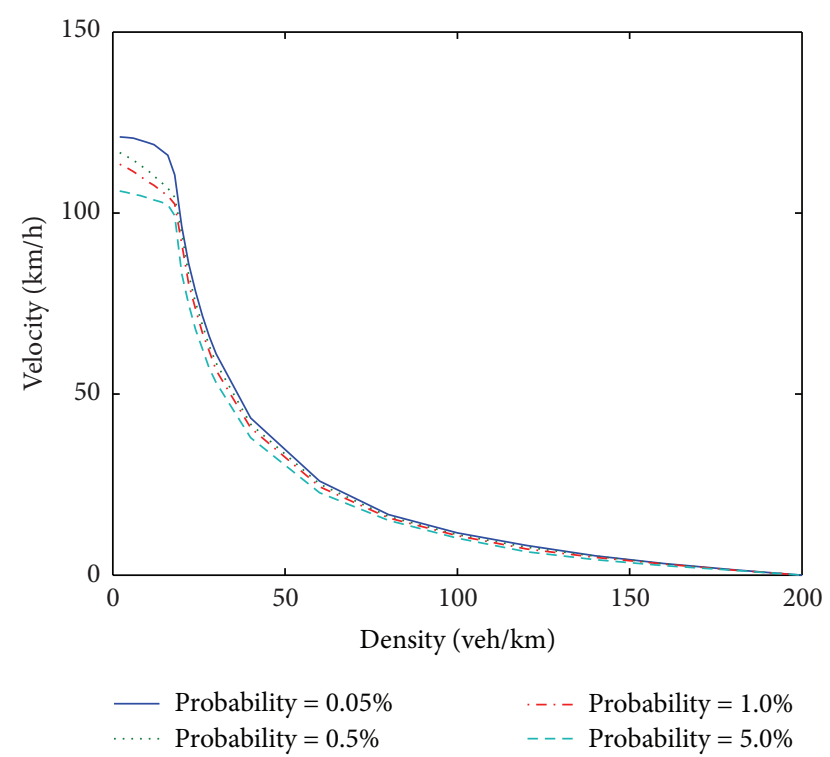

FIGURE 4: The change of velocity with different probability of phone use.

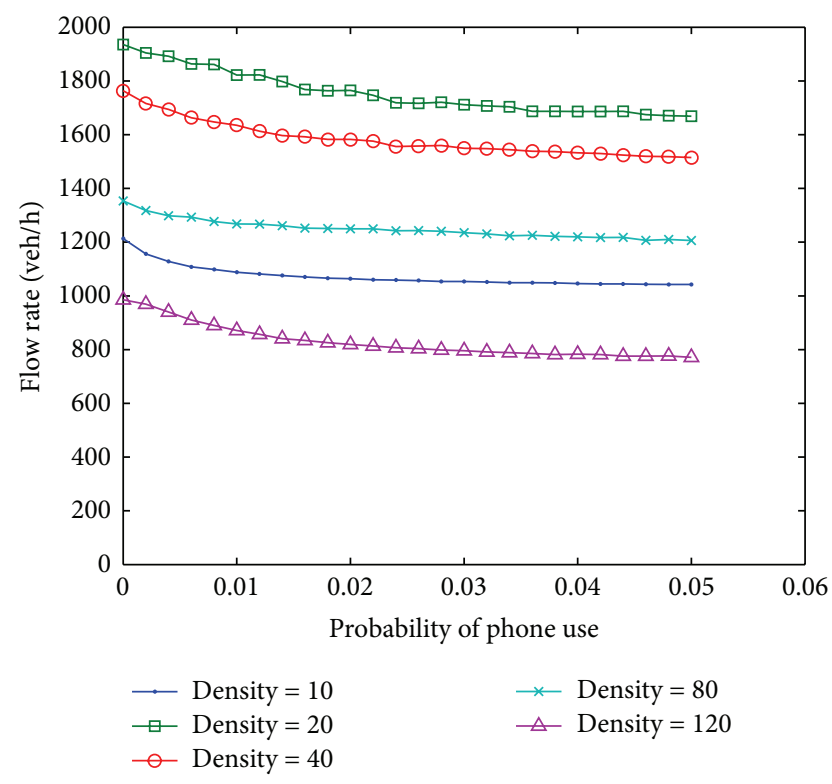

FIGURE 5: The change of flow rate under different density.

even if the proportion of distracted drivers is extremely low (approximately 2\%-3\%). That the flow rate is almost the same when density is below $10 \mathrm{veh} / \mathrm{km}$ may be due to both the low number of cars and the low probability of phone use, which means that a distracted vehicle is hardly to appear and these two situations are nearly the same.

For further research, situations with different probability of phone use were simulated and the change of flow rate was shown in Figure 3, while the change of velocity was shown in Figure 4. In order to eliminate the random effect, every situation was simulated 20 times and the average values were calculated. From the results, the changing trend of flow rate

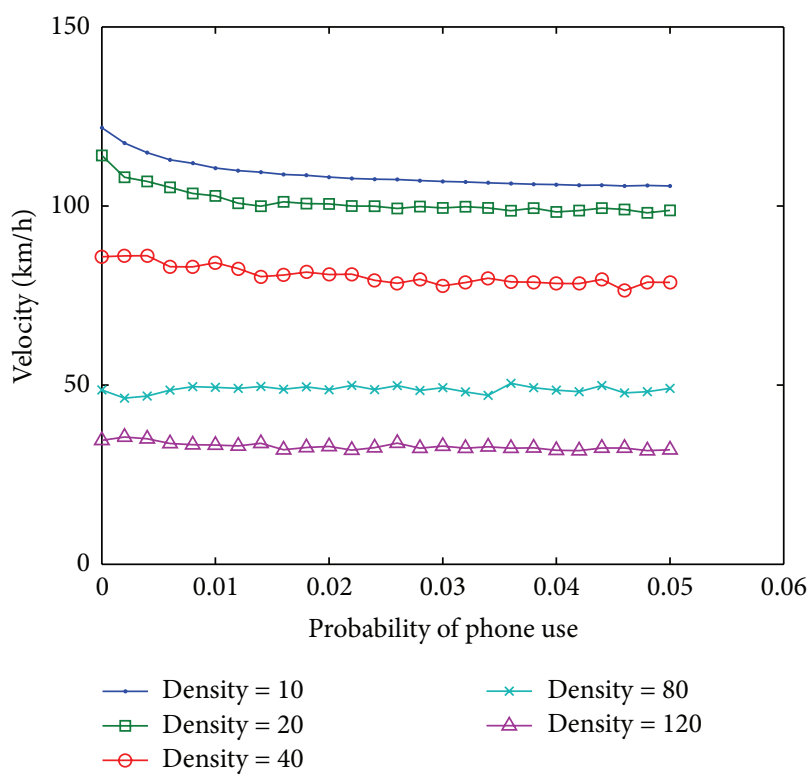

Figure 6: The change of velocity under different density.

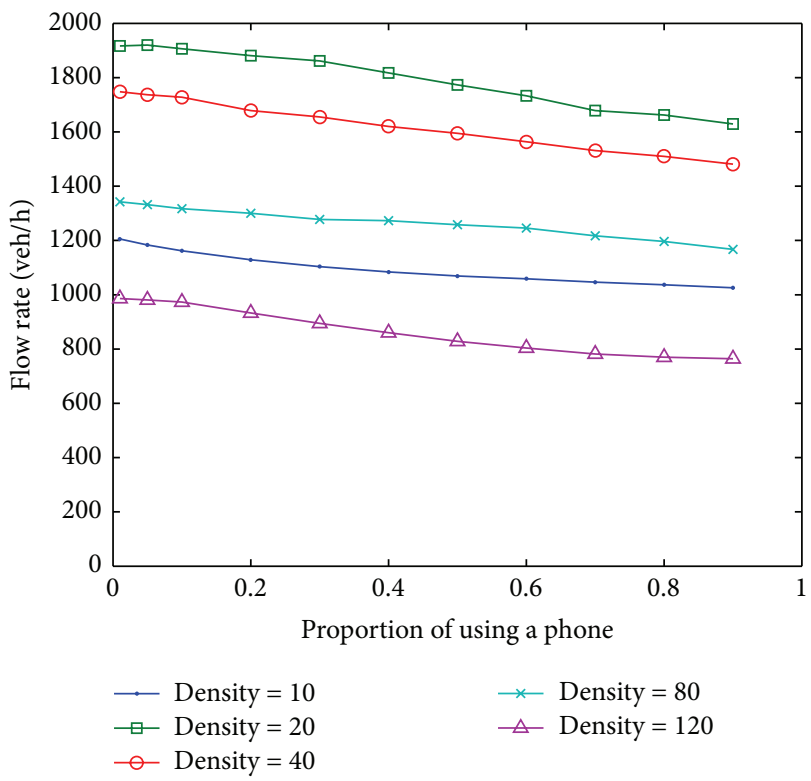

FIGURE 7: The relation between proportion of phone use $\left(p_{\text {obser }}\right)$ and flow rate.

has big differences between low density and high density as the probability of phone use increases. The maximum flow rate gradually decreases from nearly $2000 \mathrm{veh} / \mathrm{h}$ to less than $1800 \mathrm{veh} / \mathrm{h}$ as the probability of phone use increases, which may be due to the lower maximum velocity of vehicle in which driver is using a mobile phone. And under different probability of phone use, the peak of these curves, that is, the maximum flow rate, occurs at almost the same density. When the density is low, meaning that the system is in condition of free traffic, the flow rate decreases very slowly with the increase of probability of phone use under the same density. But when the density is high and the system is in congested 

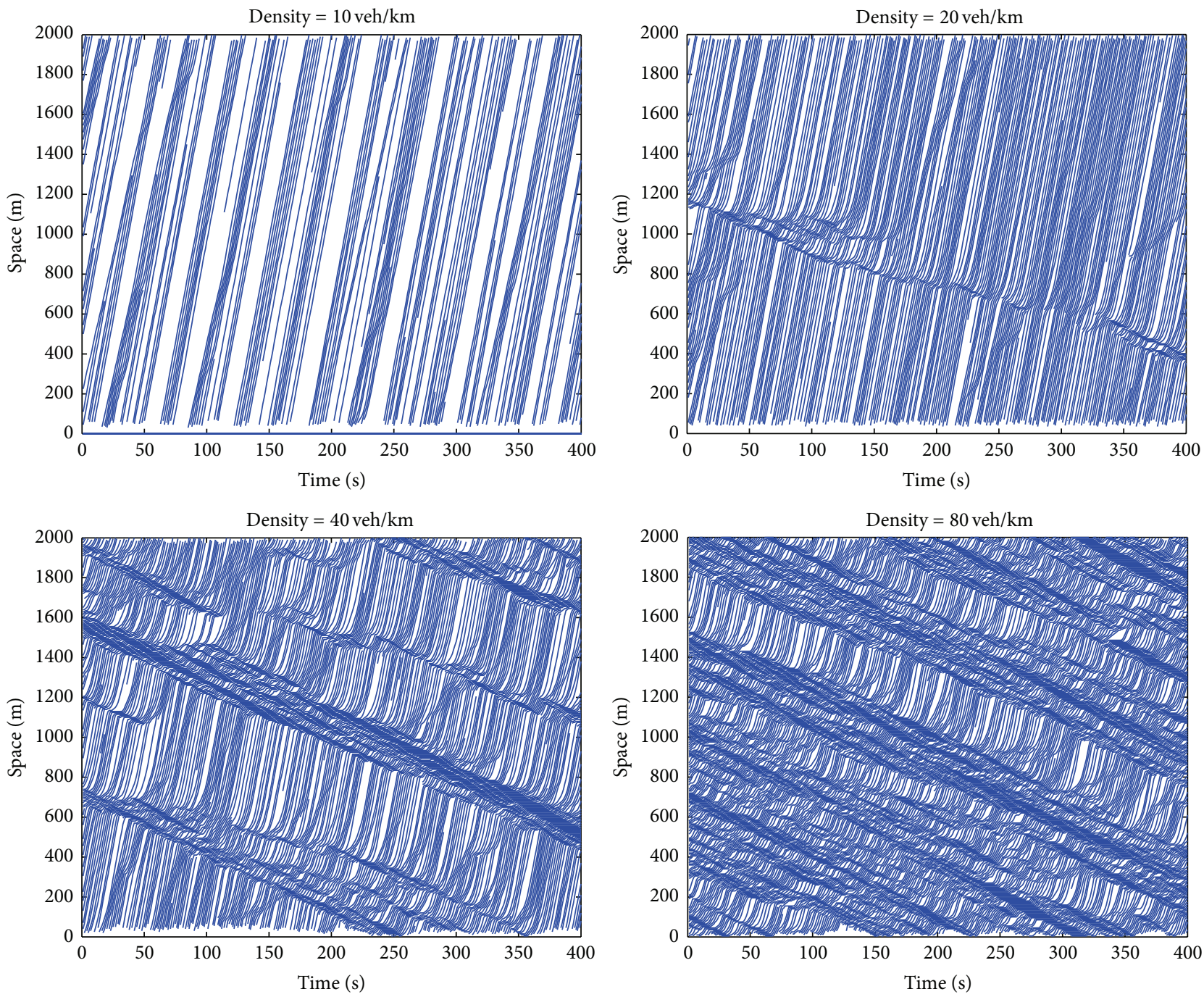

FIGURE 8: The trajectories of vehicle with different densities $\left(p_{\text {talk }}=0.05 \%\right)$.

traffic, the flow rate decreases very fast with the increase of probability of phone use. When the probability of phone use is $0.05 \%$, the maximum flow rate is the highest among the five curves. When the probability of phone use is $5 \%$, the curve is the lowest among the five curves.

The changing trend of velocity is simpler. As the density increases, the velocity continues to decrease, except that the speed of decrease becomes slow when the density reaches $50 \mathrm{veh} / \mathrm{km}$. Under low density, the monotonic velocity decreases with the increase of phone use probability. The phone use behavior of drivers has a negative influence on traffic flow. The average velocity will obviously decrease when more drivers use a phone while driving when the density is relatively low, and the change of flow rate needs to be further investigated.

3.2. The Variation of Flow Rate and Velocity. In order to analyze the influence of phone use probability on traffic flow, the average flow rate and velocity were calculated with the increase of phone use probability under a fixed density.
The simulation was also repeated 20 times to eliminate the random effect. Figures 5 and 6 show the results of simulation and the values of density are 10,20, 40, 80, and 120 .

The flow rate first significantly decreases and then almost keeps to some value with the increase of phone use probability. Whether the system is in free traffic or congested traffic, a small number of distracted drivers will disturb the traffic flow and reduce the flow rate. But when the proportion of distracted drivers is large, the more the distracted drivers occur, the more stable the traffic flow becomes, because the distracted drivers become a major part. The velocity also obviously decreases firstly and then changes little with the phone use probability under low densities. In the field research, what we observed is the proportion of drivers using a phone. So the relationship of proportion of distracted drivers and flow rate is modelling in Figure 7, based on the transition relation between proportion of phone use and probability of phone use in Figure 1.

The flow rate decreases when the proportion of drivers using a phone increases. Field investigation showed that 

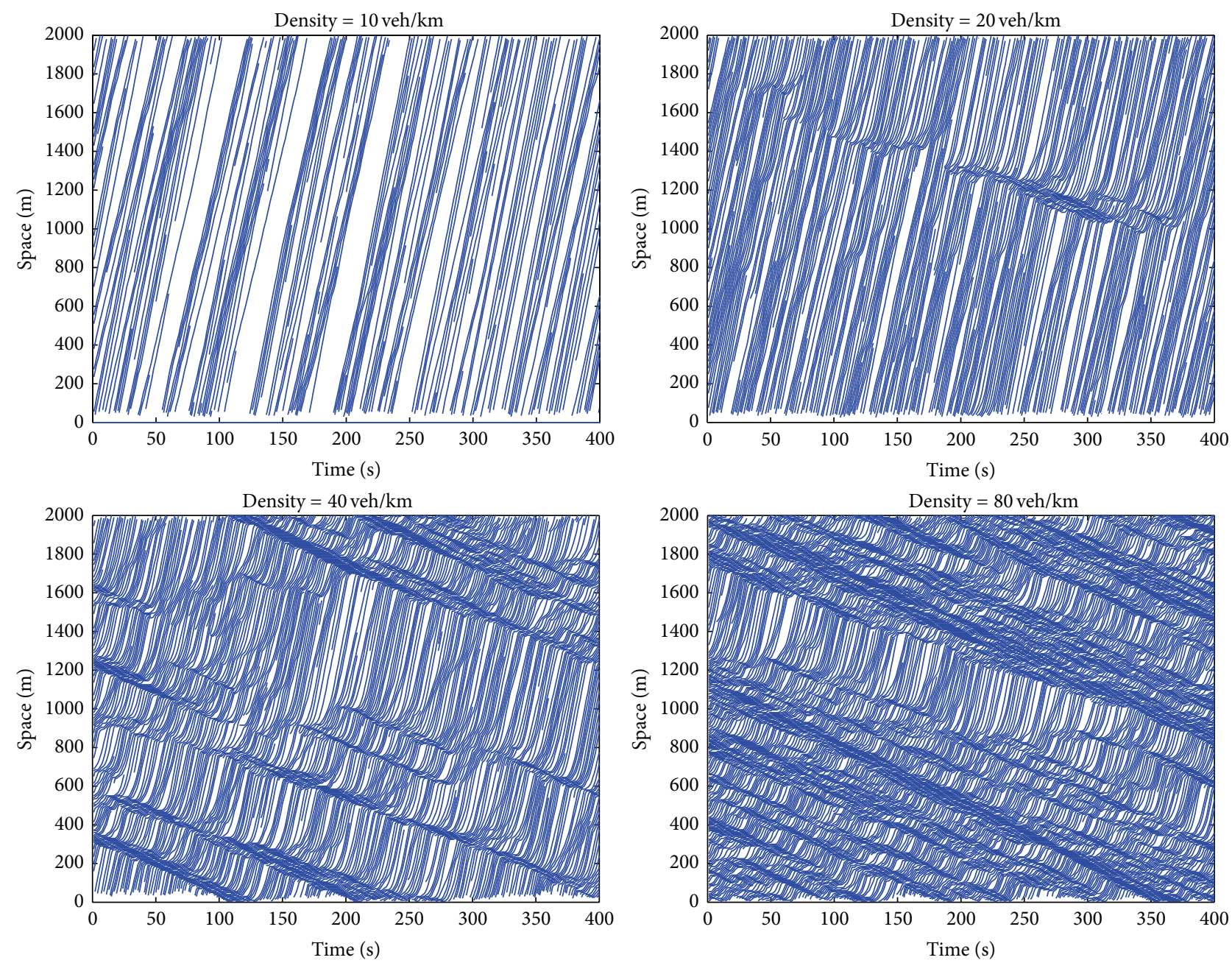

FIgURE 9: The trajectories of vehicle with different densities ( $\left.p_{\text {talk }}=0.5 \%\right)$.

the proportion of drivers using a phone ranged from $1.3 \%$ to $2.2 \%$, while the proportion of drivers who were engaged in any distracting activities was about $14.4 \%-29.8 \%$ [2, 21]. Considering that many distracted behaviors, such as manipulating music/audio controls, drinking, and smoking, may have similar impact on drivers, like reducing driving speed and the lane changing expectations, the actual traffic condition may be close to the results in Figure 7 where the proportion of distracted drivers is around $20 \%$, where the flow rate is about $5 \%$ lower than situations with no distracted drivers. Based on this hypothesis, all the distracted behaviors, including using a phone, may have reduced the flow rate by $5 \%$, according to the simulation results.

3.3. Spatiotemporal Dynamics. The patterns of spatiotemporal dynamics were also analyzed when the probability of phone use was $0.05 \%, 0.5 \%$, and $5 \%$, and the trajectories of traffic flow were shown in Figures 8-10. Given the density of $10 \mathrm{veh} / \mathrm{km}$, the distributions of space headway in Figures 8 and 10 are more homogeneous than that in Figure 9. A certain number of distracted drivers may cause bottleneck in free traffic, and the vehicles become small groups in the space-time diagram. Therefore, the flow rate is lower when distracted drivers occur.

When the density increases to $20 \mathrm{veh} / \mathrm{km}$, some small moving clusters occur and continue for only a short period of time in Figure 8. But in Figures 9 and 10, these clusters do not disappear and exist all the time. High proportion of drivers using a phone makes the clusters hard to vanish for its lower maximum velocity and higher probability to slow down. The traffic flow becomes more congested as distracted drivers increase and the condition of flow is metastable. When the density is $40 \mathrm{veh} / \mathrm{km}$ or $80 \mathrm{veh} / \mathrm{km}$, the traffic flow is in the condition of congestion and the vehicles are separated into groups of different speeds. The major factor of drivers using a phone influencing the traffic is the higher probability of slowing down, which decreases the flow rate.

3.4. Risk Analysis. Distracted behaviors can increase the likelihood of traffic accidents and damage the traffic safety. Previous studies focus on the increased risk of distracted drivers themselves to be involved in an accident, while, in 

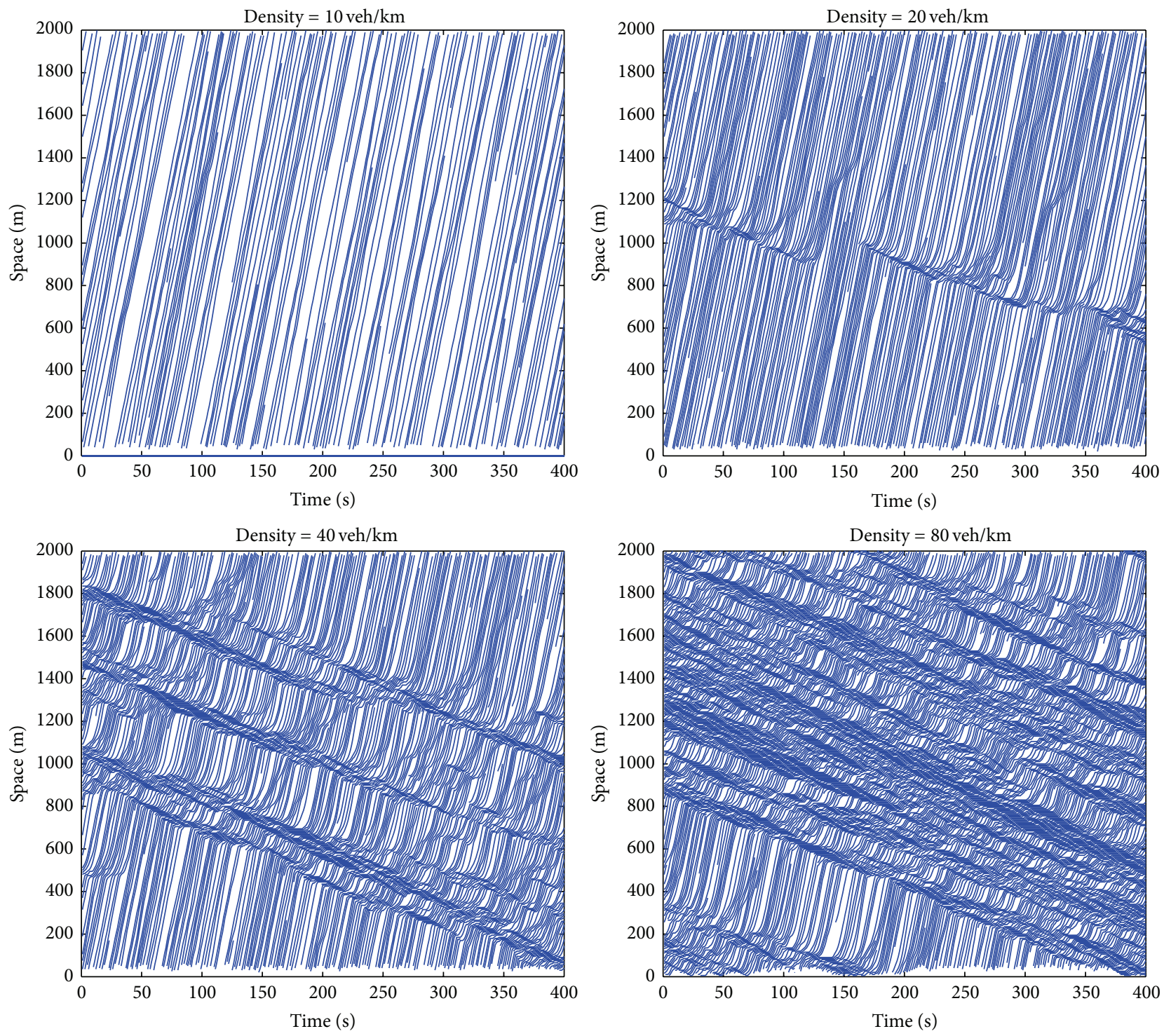

FIGURE 10: The trajectories of vehicle with different densities $\left(p_{\text {talk }}=5 \%\right)$.

this paper, the influence of distracted drivers on the safety of whole traffic was estimated. Dangerous situation was proposed to measuring the traffic safety by Boccara et al. [24] and an accident may occur if three conditions are satisfied. The empty cells between car $i$ and car $i+1$ at time $t$ is $n$, and the conditions are shown below:

$$
\begin{aligned}
0 & \leq n \leq v_{\mathrm{ds}}, \\
v(i+1, t) & >0, \\
v(i+1, t+1) & =0 .
\end{aligned}
$$

The probability of dangerous situation per vehicle per second is defined as $P_{\mathrm{ds}}$ and Shi and Tan [25] have used it to estimate the risk of traffic in heavy fog weather. For general drivers, the value of $v_{\mathrm{ds}}$ is the maximum velocity, which is 7 , while, for drivers using a phone, the value of $v_{\mathrm{ds}}$ doubles the maximum velocity of distracted vehicles $\left(v_{\max }=6\right)$, which is 12 , considering that the reaction time of distracted drivers is longer [26]. In the current paper, dangerous situation with different proportion of distracted drivers was calculated and shown in Figure 11.

The occurrence probability of dangerous situation decreases with the increase of drivers using a phone under high density. However, the risk first decreases and then increases under low density. The distracted drivers reduce the average velocity of traffic flow and the risk of the traffic; mostly the risk of undistracted drivers is lower when the proportion of distracted drivers is little. However, as the proportion of distracted drivers is large, the primary risk of the traffic comes from the distracted drivers, so the overall risk increases. When the traffic is congested, even though the driver is distracted, the risk is still lower than that in free traffic, due to the lower speed of vehicles. 

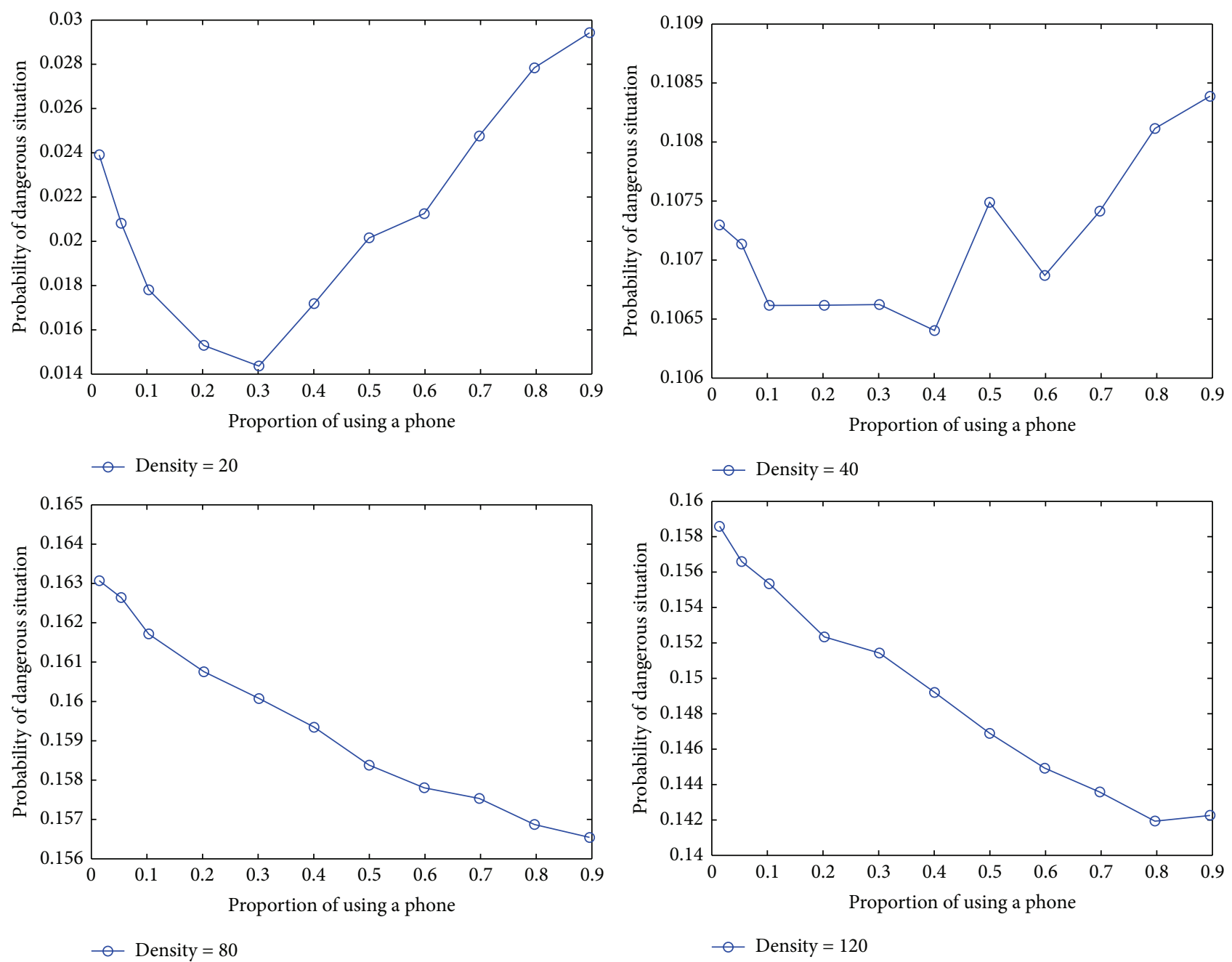

FIGURE 11: The relationship between traffic risk and phone use.

\section{Conclusion}

The focus of current paper was modelling the influence of phone use while driving on the efficiency and safety of traffic flow. A modified cellular automata model was proposed considering the phone use behaviors of drivers based on Nagel-Schreckenberg model. According to the results of simulation, even when the probability for drivers of using a phone was very low, the influence on flow rate could not be ignored. The flow rate under high density would significantly decrease whether the proportion of drivers using a phone was small or big, while the flow rate under low density would slowly decrease when the proportion of drivers using a phone was small. When the density is high, flow rate continued to decrease as the proportion increased. The average velocity gradually decreased when the proportion increased, which was more apparent at lower density. In the meantime, the distracted drivers reduce the risk of the whole traffic when the proportion is small, not considering the sharp increase of risk for themselves. And under low density, the risk of the traffic increases when the proportion of distracted drivers is large. However, this model was relatively simple and still could to be improved. The system is a $2 \mathrm{~km}$ closed circle, which makes a difference from reality. In the model, we only considered talking on a phone and the influence of texting is not studied. To obtain the comprehensive effect of phone use on traffic flow, texting should also be analyzed and discussed. We also ignored the differences among distracted drivers and every driver may show different characteristics when talking on a phone. The parameters in the model need to be further studied and calibrated. The influence of distracted behavior on traffic efficiency needed to be testified by empirical data and should be further studied.

\section{Conflict of Interests}

The authors declare that there is no conflict of interests.

\section{References}

[1] T. C. Lansdown, A. N. Stephens, and G. H. Walker, "Multiple driver distractions: a systemic transport problem," Accident Analysis \& Prevention, vol. 74, pp. 360-367, 2015. 
[2] M. J. M. Sullman, "An observational study of driver distraction in England," Transportation Research Part F: Traffic Psychology and Behaviour, vol. 15, no. 3, pp. 272-278, 2012.

[3] NHTSA, National Survey of Distracted and Drowsy Driving: Attitudes and Behaviours, Department of Transportation, Washington, DC, USA, 2002.

[4] L. Pöysti, S. Rajalin, and H. Summala, "Factors influencing the use of cellular (mobile) phone during driving and hazards while using it," Accident Analysis and Prevention, vol. 37, no. 1, pp. 4751, 2005.

[5] M. J. M. Sullman and P. H. Baas, "Mobile phone use amongst New Zealand drivers," Transportation Research Part F: Traffic Psychology and Behaviour, vol. 7, no. 2, pp. 95-105, 2004.

[6] J. S. Wang, R. R. Knipling, and M. J. Goodman, "The role of driver inattention in crashes: new statistics from the 1995 crashworthiness data system," in Proceedings of the 40th Annual Association for the Advancement of Automotive Medicine, pp. 377-392, Vancouver, Canada, 1996.

[7] S. P. McEvoy, M. R. Stevenson, and M. Woodward, "The prevalence of, and factors associated with, serious crashes involving a distracting activity," Accident Analysis and Prevention, vol. 39, no. 3, pp. 475-482, 2007.

[8] V. K. Lee, C. R. Champagne, and L. H. Francescutti, "Fatal distraction: cell phone use while driving," Canadian Family Physician, vol. 59, no. 7, pp. 723-725, 2013.

[9] S. P. McEvoy, M. R. Stevenson, A. T. McCartt et al., "Role of mobile phones in motor vehicle crashes resulting in hospital attendance: a case-crossover study," British Medical Journal, vol. 331, no. 7514, pp. 428-430, 2005.

[10] K. Nagel and M. Schreckenberg, "A cellular automaton model for freeway traffic," Journal de Physique I, vol. 2, no. 12, pp. 22212229, 1992.

[11] Y. S. Qian, X. M. Shao, J. W. Zeng, and M. Wang, "An improved cellular automaton model with the consideration of a multi-point tollbooth," Physica A: Statistical Mechanics and its Applications, vol. 392, no. 23, pp. 5874-5878, 2013.

[12] C. Chai and Y. D. Wong, "Micro-simulation of vehicle conflicts involving right-turn vehicles at signalized intersections based on cellular automata," Accident Analysis and Prevention, vol. 63, pp. 94-103, 2014.

[13] J. Esser and M. Schreckenberg, "Microscopic simulation of urban traffic based on cellular automata," International Journal of Modern Physics C, vol. 8, no. 5, pp. 1025-1036, 1997.

[14] P. M. Simon and K. Nagel, "Simplified cellular automaton model for city traffic," Physical Review E-Statistical Physics, Plasmas, Fluids, and Related Interdisciplinary Topics, vol. 58, no. 2, pp. 1286-1295, 1998.

[15] G. Diedrich, L. Santen, A. Schadschneider, and J. Zittartz, "Effects of on- and off-ramps in cellular automata models for traffic flow," International Journal of Modern Physics C, vol. 11, no. 2, pp. 335-345, 2000.

[16] J. Vasic and H. J. Ruskin, "Cellular automata simulation of traffic including cars and bicycles," Physica A: Statistical Mechanics and Its Applications, vol. 391, no. 8, pp. 2720-2729, 2012.

[17] R. Jiang and Q.-S. Wu, "A stopped time dependent randomization cellular automata model for traffic flow controlled by traffic light," Physica A: Statistical Mechanics and Its Applications, vol. 364, pp. 493-496, 2006.

[18] M. Rickert, K. Nagel, M. Schreckenberg, and A. Latour, "Two lane traffic simulations using cellular automata," Physica A: Statistical Mechanics and its Applications, vol. 231, no. 4, pp. 534550, 1996.
[19] W. J. Horrey and C. D. Wickens, "Examining the impact of cell phone conversations on driving using meta-analytic techniques," Human Factors, vol. 48, no. 1, pp. 196-205, 2006.

[20] J. M. Cooper, I. Vladisavljevic, N. Medeiros-Ward, P. T. Martin, and D. L. Strayer, "An investigation of driver distraction near the tipping point of traffic flow stability," Human Factors, vol. 51, no. 2, pp. 261-268, 2009.

[21] J. Stutts, J. Feaganes, D. Reinfurt et al., "Driver's exposure to distractions in their natural driving environment," Accident Analysis \& Prevention, vol. 37, no. 6, pp. 1093-1101, 2005.

[22] P. Simon and H. Gutowitz, "Cellular automaton model for bidirectional traffic," Physical Review E, vol. 57, no. 2, pp. 24412444, 1998.

[23] D. Stavrinos, J. L. Jones, A. A. Garner et al., "Impact of distracted driving on safety and traffic flow," Accident Analysis and Prevention, vol. 61, pp. 63-70, 2013.

[24] N. Boccara, H. Fuks, and Q. Zeng, "Car accidents and number of stopped cars due to road blockage on a one-lane highway," Journal of Physics A: Mathematical and General, vol. 30, no. 10, pp. 3329-3332, 1997.

[25] J. Shi and J. Tan, "Effect analysis of intermittent release measures in heavy fog weather with an improved CA model," Discrete Dynamics in Nature and Society, vol. 2013, Article ID 812562, 7 pages, 2013.

[26] M. M. Haque and S. Washington, "A parametric duration model of the reaction times of drivers distracted by mobile phone conversations," Accident Analysis and Prevention, vol. 62, pp. 42-53, 2014. 


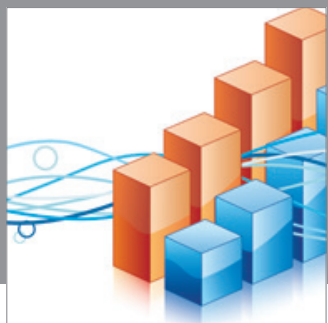

Advances in

Operations Research

mansans

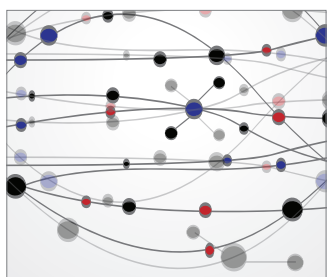

The Scientific World Journal
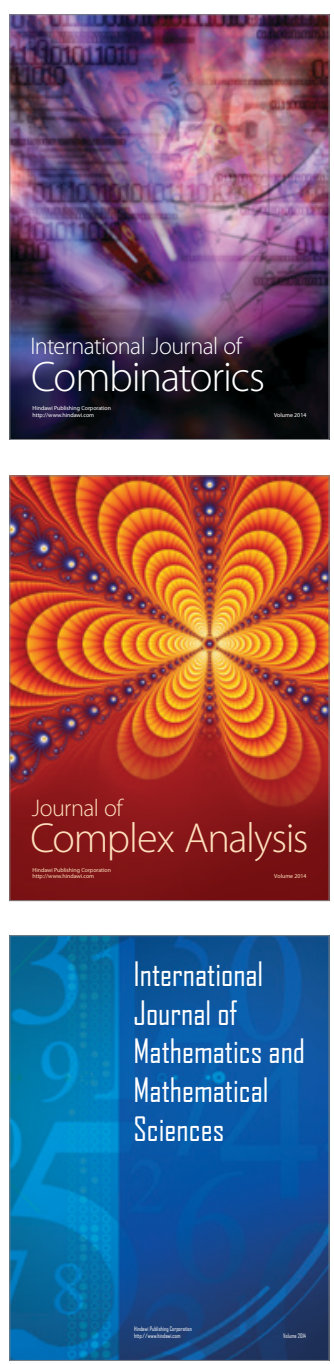
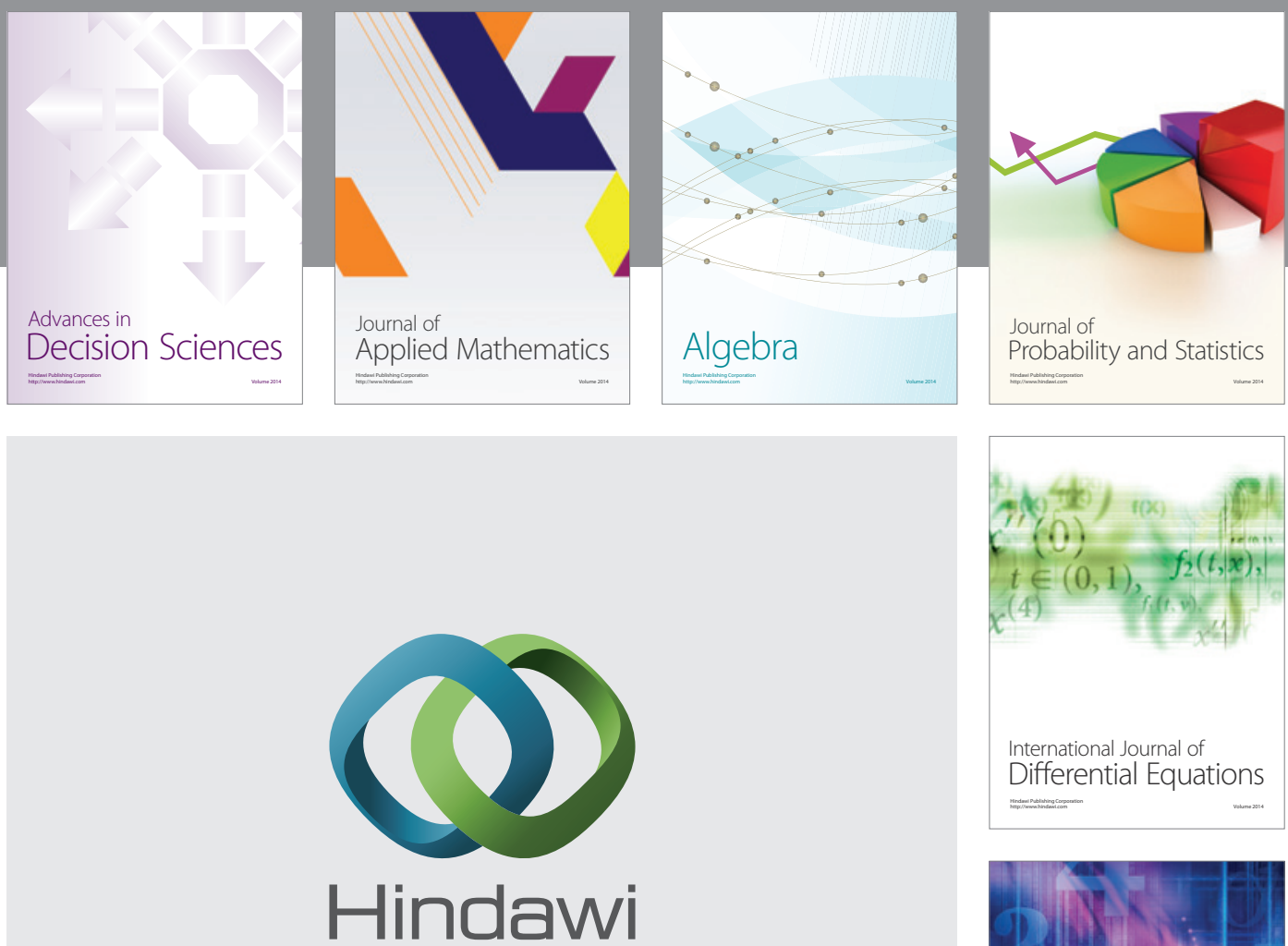

Submit your manuscripts at http://www.hindawi.com
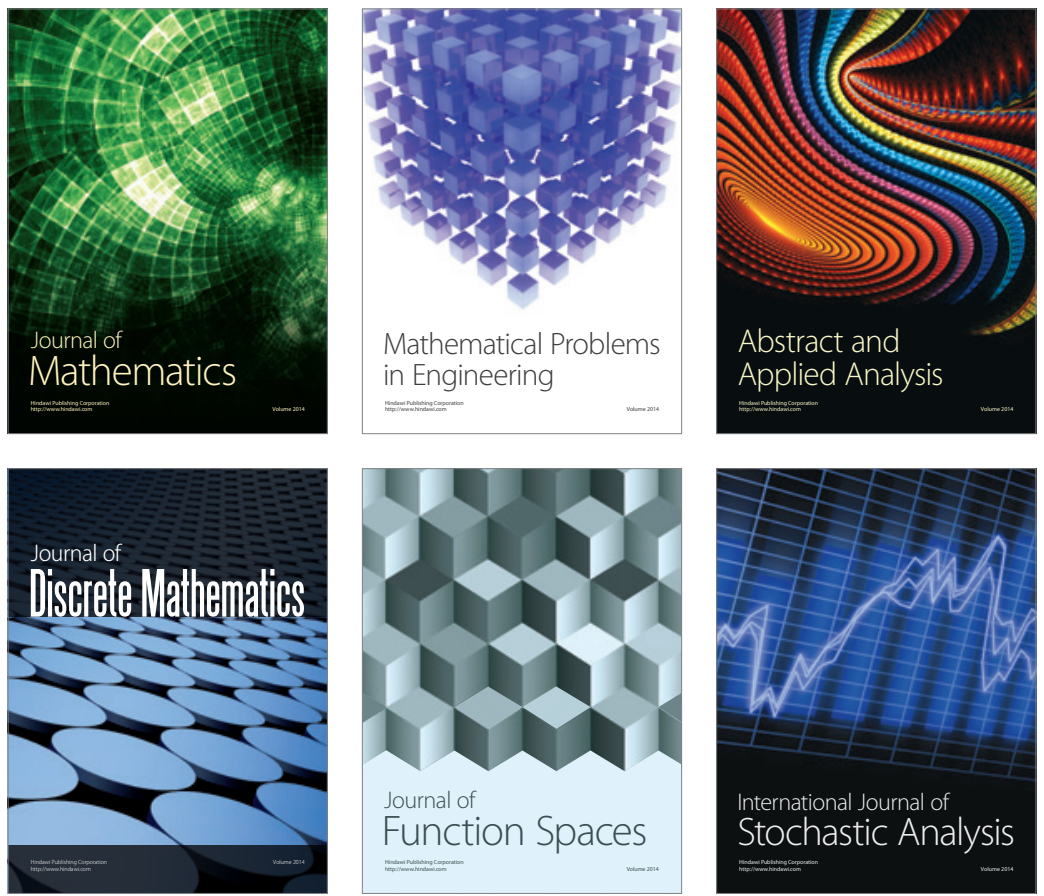

Journal of

Function Spaces

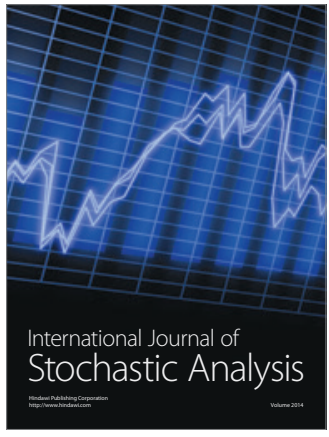

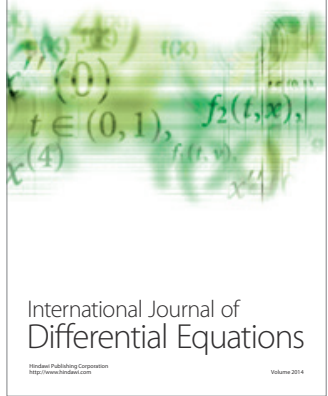
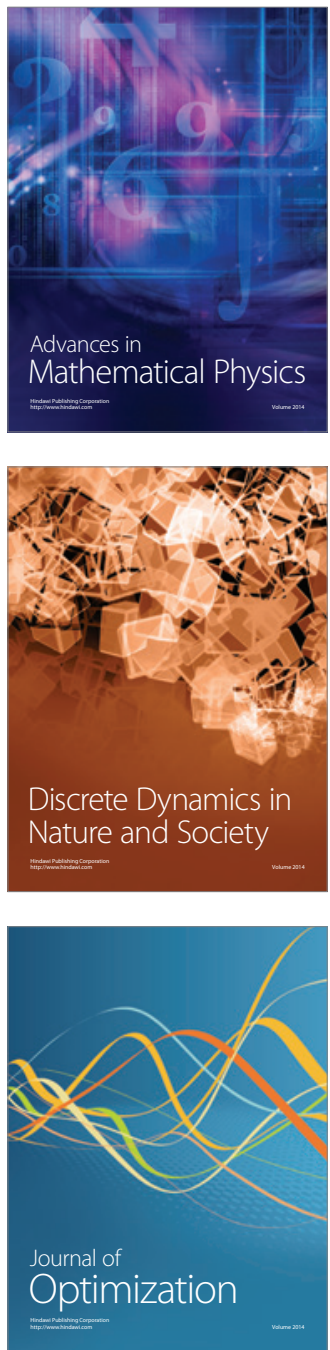